Quel type de professions scientifiques les garçons et les filles de 15 ans envisagent-ils d'exercer?

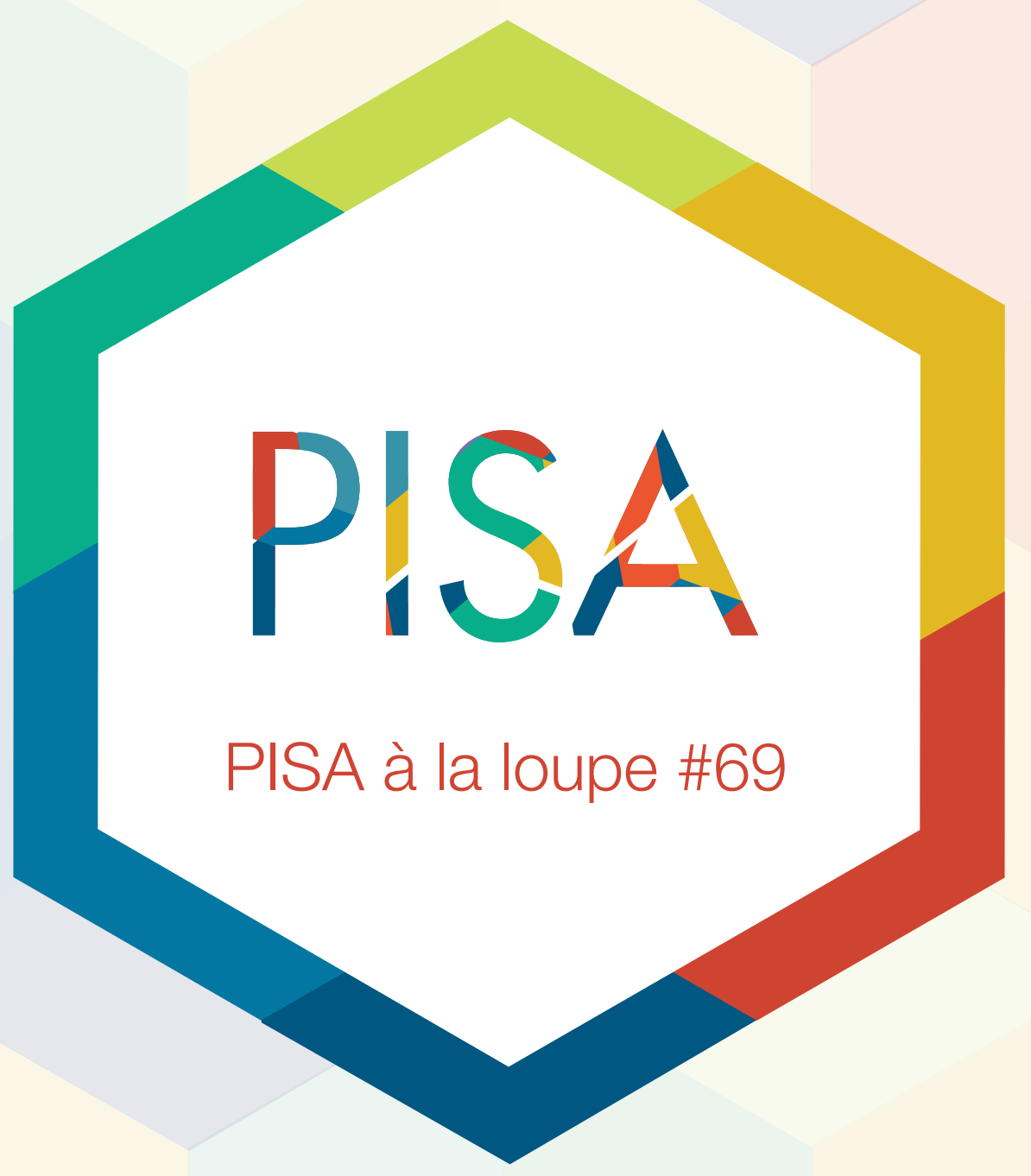




\section{Quel type de professions scientifiques les garçons et les filles de 15 ans envisagent-ils d'exercer?}

- En moyenne, dans les pays de l'OCDE, près d'un élève sur quatre - garçons et filles confondus - envisage d'exercer une profession nécessitant de suivre une formation plus poussée en sciences après la scolarité obligatoire.

- Les garçons sont plus de deux fois plus susceptibles que les filles d'envisager d'exercer le métier d'ingénieur, de scientifique ou d'architecte. En outre, en moyenne, dans les pays de I'OCDE, $4.8 \%$ des garçons, contre seulement $0.4 \%$ des filles, envisagent d'exercer une profession dans le domaine des TIC.

- Les filles sont près de trois fois plus susceptibles que les garçons d'envisager d'exercer le métier de médecin, de vétérinaire, de cadre infirmier ou autre professionnel de santé.

Si vous avez récemment eu l'occasion de vous rendre dans un hôpital, il y a fort à parier que le personnel qui vous a reçu - non seulement infirmier, mais aussi médical - était majoritairement féminin. L'augmentation rapide du pourcentage de femmes médecins montre que même les idées reçues les plus tenaces sur les rôles traditionnellement dévolus aux hommes et aux femmes peuvent vite évoluer. II y a seulement une génération, les femmes ne représentaient qu'une minorité des médecins dans la plupart des pays. Elles n'ont toutefois pas enregistré les mêmes progrès dans toutes les professions scientifiques et sont par exemple très peu nombreuses à occuper des postes universitaires de haut niveau en physique, discipline dans laquelle le dernier prix Nobel décerné à une femme remonte à 1963. Parallèlement, les nouvelles professions du secteur émergent des technologies de l'information et de la communication sont souvent, et presque exclusivement, dominées par les hommes.

La ségrégation professionnelle - le fait que les hommes et les femmes exercent des professions différentes, même dans des domaines étroitement liés - est l'une des principales causes de la persistance des écarts salariaux entre les sexes. Les pays qui encouragent de façon identique les garçons et les filles à exercer une profession scientifique peuvent non seulement réduire les écarts salariaux entre les sexes, mais aussi garantir qu'aucun talent porteur d'innovation et de croissance ne se perde, et ce, pour le bénéfice de tous.

En 2015, l'enquête PISA demandait aux élèves quelle profession ils envisageaient d'exercer à l'âge de 30 ans. Leurs réponses ont ensuite été regroupées en deux grandes catégories (professions scientifiques ou non), la première incluant les spécialistes des sciences techniques, les spécialistes de la santé, les professions intermédiaires à caractère scientifique et les spécialistes des technologies de l'information et des communications (TIC).

\section{Filles et garçons sont presque aussi susceptibles d'envisager d'exercer une profession scientifique.}

En moyenne, dans les pays de l'OCDE, près d'un élève sur quatre (24 \%) déclare envisager d'exercer une profession nécessitant de suivre une formation plus poussée en sciences après la scolarité obligatoire. Plus spécifiquement, ils sont $8.6 \%$ à envisager d'exercer une profession de spécialiste des sciences techniques (par exemple, ingénieur, architecte, physicien ou astronome), $11.4 \%$ une profession de spécialiste de la santé (par exemple, médecin, cadre infirmier, vétérinaire ou physiothérapeute), $2.6 \%$ une profession de spécialiste des technologies de l'information et des communications (par exemple, concepteur de logiciels ou programmeur d'applications) et $1.4 \%$ une profession intermédiaire à caractère scientifique (par exemple, technicien de télécommunications ou en électricité). 
En moyenne, dans les pays de l'OCDE, garçons et filles sont presque aussi susceptibles d'envisager une carrière dans un domaine scientifique : $25 \%$ des garçons se disent dans ce cas, contre $24 \%$ des filles. Toutefois, même lorsque les pourcentages de garçons et de filles espérant exercer une profession scientifique sont équilibrés, les uns et les autres n'envisagent pas les mêmes carrières.

\section{Aspirations à embrasser une carrière scientifique chez les garçons et les filles}

\section{Moyenne OCDE}

Élèves envisageant d'exercer..

- ...une profession de spécialiste des sciences techniques

-...une profession de spécialiste de la santé

- ...une profession de spécialiste des technologies de l'information

...une profession intermédiaire à caractère scientifique et des communications (TIC)

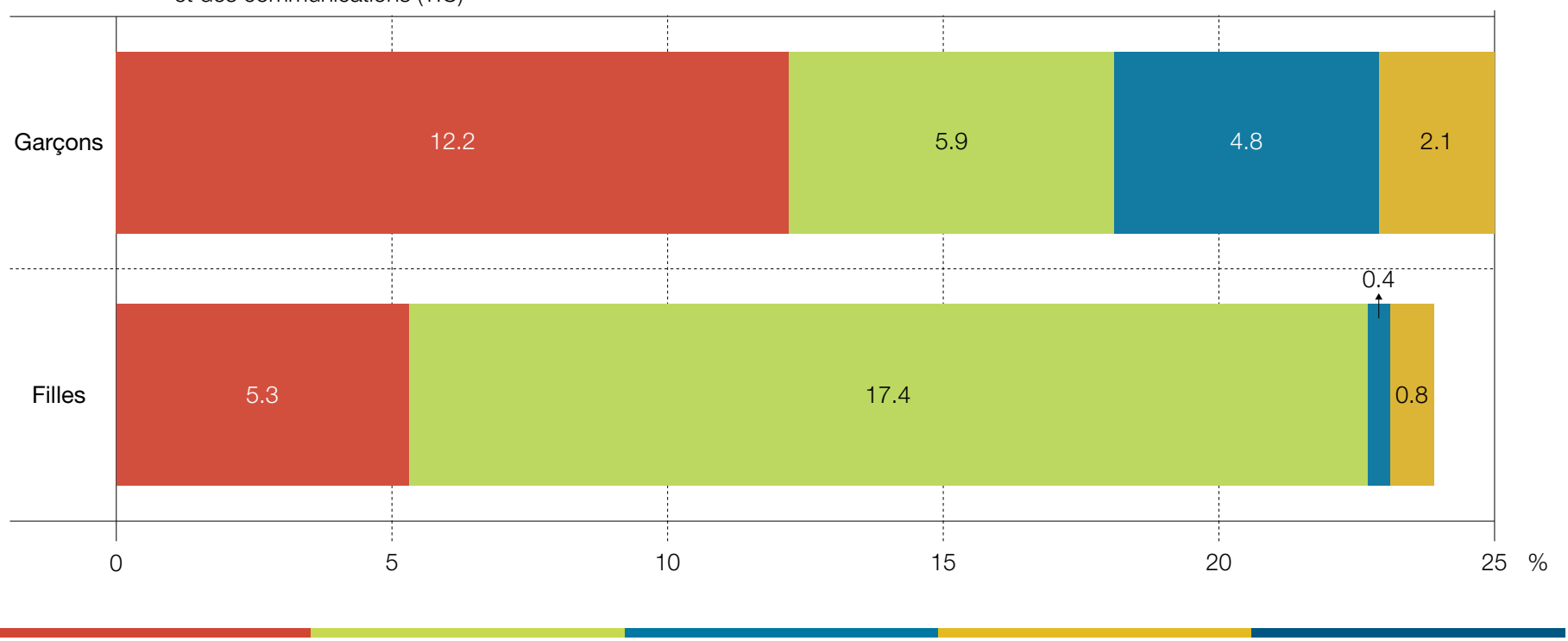

Source : OCDE, Base de données PISA 2015, tableaux I.3.11a-d.

Statlink : http://dx.doi.org/10.1787/888933432311

Cependant, garçons et filles divergent de par leurs centres d'intérêts et leurs aspirations pour leur future carrière scientifique.

Les filles sont trois fois plus susceptibles que les garçons d'envisager d'exercer le métier de médecin, de vétérinaire ou de cadre infirmier. Si la profession de médecin est autant prisée par les garçons que par les filles, les autres professions du secteur de la santé sont rarement plébiscitées dans les aspirations professionnelles des garçons, alors qu'elles comptent parmi les plus populaires chez les filles. II s'agit des professions de dentiste, pharmacien, physiothérapeute, diététicien, cadre infirmier, sage-femme et vétérinaire.

Parallèlement, les garçons envisagent davantage que les filles d'exercer le métier de spécialiste des TIC, de scientifique ou d'ingénieur. Si nombre de filles peuvent envisager d'exercer la profession d'architecte ou de concepteur, elles sont bien moins nombreuses à aspirer à une carrière d'ingénieur ou de concepteur de logiciels. En moyenne, dans les pays de l'OCDE, seuls $0.4 \%$ des filles, contre $4.8 \%$ des garçons, envisagent ainsi d'exercer une profession de spécialiste des TIC.

Si les données de l'enquête PISA ne dévoilent que les aspirations professionnelles des jeunes de 15 ans - tout à fait susceptibles de changer avant les choix décisifs de carrière -, les différences d'aspirations entre les garçons et les filles à l'âge de 15 ans correspondent bien aux tendances récentes observées dans les inscriptions en licence dans l'enseignement tertiaire. En 2013, en moyenne, dans les pays de l'OCDE, les femmes représentaient 78 \% des nouveaux inscrits dans des formations en santé et protection sociale, contre seulement $30 \%$ en sciences et en ingénierie. 


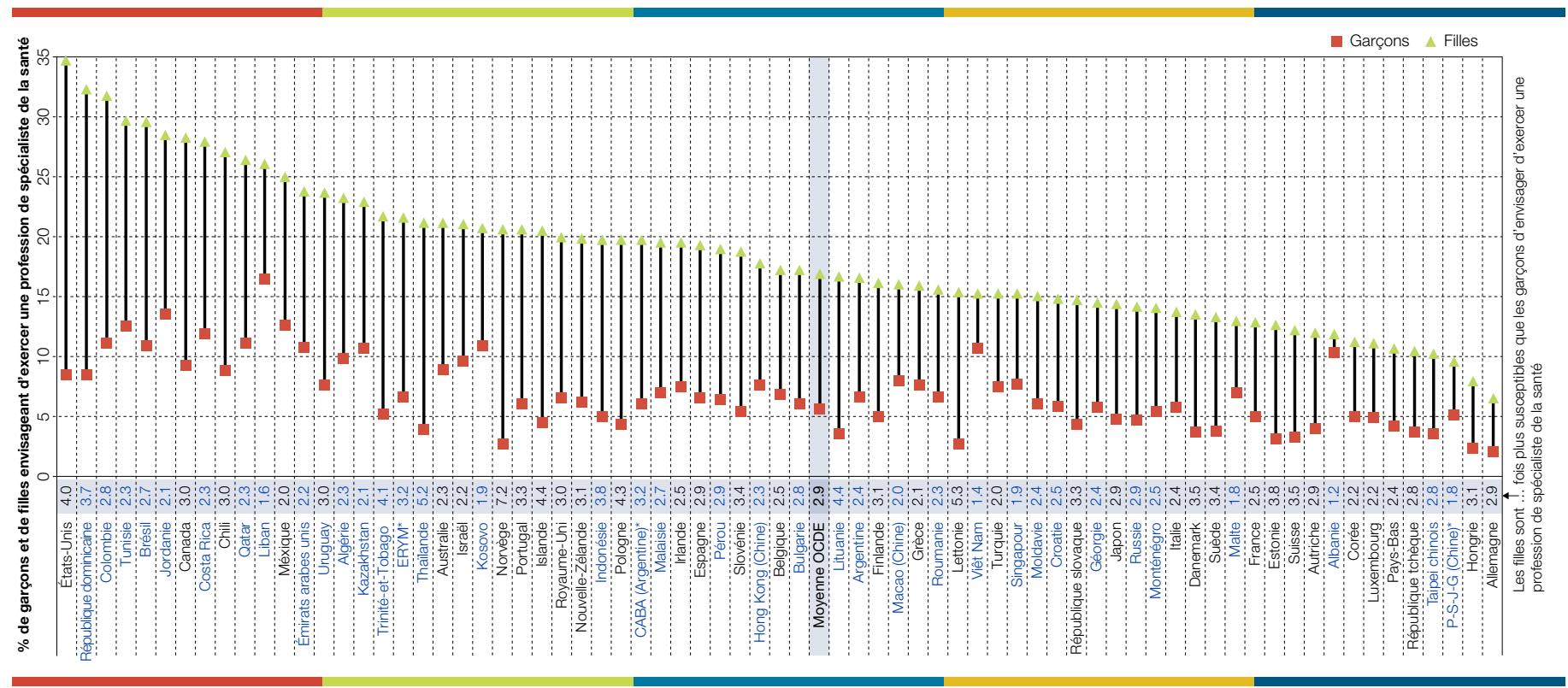

*Les acronymes P-S-J-G (Chine), CABA et ERYM désignent respectivement : les quatre provinces chinoises participant à l'enquête PISA (Pékin, Shanghai, Jiangsu et Guangdong), la Ciudad Autónoma de Buenos Aires (Argentine) et l'ex-République yougoslave de Macédoine.

Remarque : L'augmentation de la probabilité (risque relatif) pour les filles d'envisager d'exercer une profession de spécialiste de la santé est indiquée en regard du nom du pays/de l'économie ; l'utilisation du symbole « n.s. » indique que le risque relatif ne s'écarte pas de 1 dans une mesure statistiquement significative (probabilité identique).

Les pays et économies sont classés par ordre décroissant du pourcentage de filles envisageant d'exercer une profession de spécialiste de la santé.

Source : OCDE, Base de données PISA 2015, tableau I.3.11b.

Statlink : http://dx.doi.org/10.1787/888933433183

Les ingénieurs et les scientifiques viennent de Mars (pour le moment)

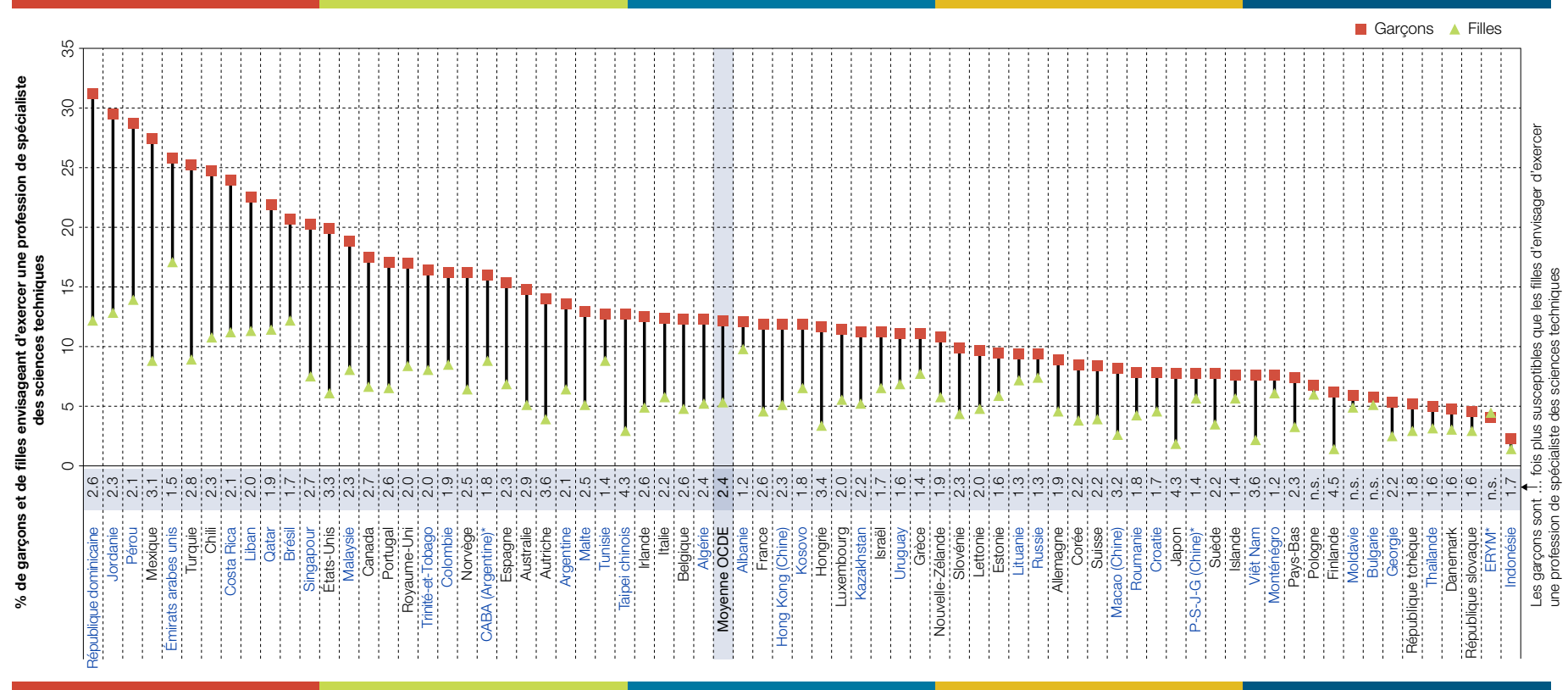

*Les acronymes P-S-J-G (Chine), CABA et ERYM désignent respectivement : les quatre provinces chinoises participant à l'enquête PISA (Pékin, Shanghai Jiangsu et Guangdong), la Ciudad Autónoma de Buenos Aires (Argentine) et l'ex-République yougoslave de Macédoine.

Remarque : L'augmentation de la probabilité (risque relatif) pour les garçons d'envisager d'exercer une profession de spécialiste des sciences techniques est indiquée en regard du nom du pays/de l'économie ; l'utilisation du symbole "n.s. » indique que le risque relatif ne s'écarte pas de 1 dans une mesure statistiquement significative (probabilité identique).

Les pays et économies sont classés par ordre décroissant du pourcentage de garçons envisageant d'exercer une profession de spécialiste des sciences techniques.

Source : OCDE, Base de données PISA 2015, tableau I.3.11a.

Statlink : http://dx.doi.org/10.1787/888933433183 
Dans certains pays, les disparités d'aspirations professionnelles sont particulièrement marquées entre les garçons et les filles. En Norvège, par exemple, si les garçons sont $29 \%$, et les filles $28 \%$, à espérer embrasser une carrière scientifique, ces dernières sont sept fois plus nombreuses (21\% contre $3 \%$ ) à envisager d'exercer le métier de médecin, de cadre infirmier ou autre spécialiste de la santé. En Finlande, les garçons sont plus de quatre fois plus susceptibles que les filles d'envisager d'exercer la profession d'ingénieur, de scientifique ou d'architecte (6.1\% contre $1.4 \%$ ), alors que les filles sont plus de trois fois plus susceptibles d'envisager une carrière de spécialiste de la santé (16\% contre $5 \%$ ).

Ces différences d'aspirations professionnelles entre les garçons et les filles reflètent dans une certaine mesure leurs différences de centres d'intérêt. D'après les données de l'enquête PISA, les garçons manifestent ainsi plus d'intérêt que les filles pour les sciences, en général, et pour les sujets en lien avec la physique et la chimie, en particulier, alors que les filles sont globalement plus attirées par les sujets liés à la santé. Toutefois, les aspirations à exercer une profession scientifique diffèrent également souvent entre des garçons et des filles tout aussi intéressés par les sciences et capables d'obtenir d'excellents résultats aux épreuves PISA dans ce domaine. En Allemagne, en Hongrie et en Suède, par exemple, les garçons se situant au moins au niveau 5 de compétence en sciences (soit les garçons les plus performants) sont significativement plus susceptibles que les filles les plus performantes d'envisager d'exercer une profession nécessitant une formation plus approfondie en sciences. Ce constat corrobore les résultats d'autres études d'après lesquels nombre d'élèves disent aimer les sciences, mais affirment ne pas se sentir faits pour ce domaine.

\section{Pour conclure}

Sous l'influence de leur famille et de la culture populaire, les filles se représentent souvent les scientifiques comme des hommes en blouse blanche, l'informatique comme un domaine " masculin », et la réussite en sciences comme résultant de quelque don intellectuel - dont elles s'estiment trop souvent dépourvues - plutôt que d'un travail assidu. Ce type de stéréotypes, quand bien même ils contiendraient une part de vérité, dissuadent des jeunes femmes tout à fait talentueuses et intéressées par les sciences d'envisager d'exercer un certain nombre de professions en lien avec les sciences, la technologie ou l'ingénierie.

L'école peut combattre ces stéréotypes et aider les élèves à embrasser une vision plus inclusive des sciences, en leur dispensant de meilleures informations en matière d'orientation professionnelle. Les élèves doivent avoir accès à des informations pertinentes, crédibles et évitant les représentations irréalistes ou caricaturales des différents débouchés professionnels. Les employeurs et les formateurs de domaines perçus comme typiquement « masculins » ou « féminins » ont aussi la possibilité d'aider à venir à bout des stéréotypes existants, en montrant par exemple aux élèves que l'informatique (domaine « masculin » et " nerd ») aide à résoudre les problèmes de santé (domaine " féminin » et " altruiste ") ou en communiquant et en établissant un contact direct avec les élèves et les établissements. Enfin, les enseignants peuvent jouer un rôle déterminant en cultivant l'intérêt des garçons comme des filles pour un large éventail de sujets scientifiques. 


\section{Pour tout complément d'information}

Contacter : Francesco Avvisati (francesco.avvisati@oecd.org)

Consulter : OCDE (2016), Résultats du PISA 2015 (Volume I) : L'excellence et l'équité dans l'éducation, PISA, Éditions OCDE, Paris, https://doi.org/10.1787/9789264267534-fr.

\section{Voir}

www. oecd.org/pisa

www.oecd-ilibrary.org/education/pisa-a-la-loupe

Les compétences des adultes à la loupe

Les indicateurs de l'éducation à la loupe

L'enseignement à la loupe

Découvrez les résultats du PISA 2015 par pays : www.compareyourcountry.org/pisa

Le mois prochain : Sélection et développement professionnel des enseignants : que nous apprennent les pays très performants?

Ce document est publié sous la responsabilité du Secrétaire général de l'OCDE. Les opinions et les arguments exprimés ici ne reflètent pas nécessairement les vues officielles des pays membres de l'OCDE.

Ce document, ainsi que les données et cartes qu'il peut comprendre, sont sans préjudice du statut de tout territoire, de la souveraineté s'exerçant sur ce dernier, du tracé des frontières et limites internationales, et du nom de tout territoire, ville ou région.

Les données statistiques concernant Israël sont fournies par et sous la responsabilité des autorités israéliennes compétentes.

L'utilisation de ces données par l'OCDE est sans préjudice du statut des hauteurs du Golan, de Jérusalem Est et des colonies de peuplement israéliennes en Cisjordanie aux termes du droit international

Ce texte est disponible sous licence Attribution - Pas d'Utilisation Commerciale - Partage dans les Mêmes Conditions 3.0 Organisations Internationales (CC BY-NC-SA 3.0 IGO).Pour toute information spécifique quant à l'étendue et aux termes de la licence ainsi que d'une possible utilisation commerciale de ce texte ou pour tout usage de données PISA, prière de consulter les Conditions d'utilisation sur www.oecd.org/fr/conditionsdutilisation. 